\title{
Symmetry Breakdown in Franckeite: Spontaneous Strain, Rippling, and Interlayer Moiré
}

Riccardo Frisenda, ${ }^{*, \#}$ Gabriel Sanchez-Santolino, ${ }^{*, \#}$ Nikos Papadopoulos, Joanna Urban, Michal Baranowski, Alessandro Surrente, Duncan K. Maude, Mar Garcia-Hernandez, Herre S. J. van der Zant, Paulina Plochocka,* Pablo San-Jose,* and Andres Castellanos-Gomez*

Cite This: Nano Lett. 2020, 20, 1141-1147

ABSTRACT: Franckeite is a naturally occurring layered mineral with a structure composed of alternating stacks of $\mathrm{SnS}_{2}$-like and $\mathrm{PbS}$-like layers. Although this superlattice is composed of a sequence of isotropic twodimensional layers, it exhibits a spontaneous rippling that makes the material structurally anisotropic. We demonstrate that this rippling comes hand in hand with an inhomogeneous in-plane strain profile and anisotropic electrical, vibrational, and optical properties. We argue that this symmetry breakdown results from a spatial modulation of the van der Waals interaction between layers due to the $\mathrm{SnS}_{2}$-like and $\mathrm{PbS}$-like lattices incommensurability.
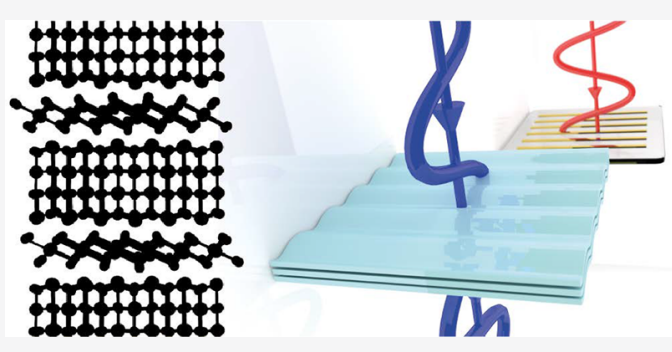

KEYWORDS: 2D material, franckeite, strain, interlayer moire, anisotropic material

\section{INTRODUCTION}

Since the isolation of graphene and other two-dimensional (2D) materials by mechanical exfoliation ${ }^{1,2}$ a large fraction of the community working on this family of materials turned their attention toward the possibility of fabricating artificial heterostructures and superlattices by stacking dissimilar $2 \mathrm{D}$ materials on top of each other. ${ }^{3-8}$ These stacks can show electronic and optical properties that strongly differ from those of the constituent $2 \mathrm{D}$ materials, thus opening the door to the fabrication of materials with user-designed properties. The most extended method to fabricate $2 \mathrm{D}$ heterostructures and superlattices consists on the manual or robotic assembly of the stacks using deterministic placement methods. ${ }^{9-12}$ Alternatively to those top down approaches, it has been recently shown that van der Waals superlattices can be naturally found in certain layered minerals, like the sulfosalts franckeite and cylindrite, that overcome a thermodynamic phase separation during their growth. ${ }^{13-16}$ In fact, franckeite and cylindrite are layered minerals with a structure composed of alternating stacks of $\mathrm{SnS}_{2}$-like pseudohexagonal $(\mathrm{H})$ and $\mathrm{PbS}$-like pseudotetragonal (Q) layers. ${ }^{17}$ Franckeite has been exfoliated (mechanically and by liquid phase exfoliation) down to the single unit cell and the exfoliated flakes have been assembled into electronic devices and photodetectors operating in the near-infrared range. ${ }^{13,14,18-21}$

Here we present a study of an intriguing feature of franckeite: although the crystal is composed of isotropic $2 \mathrm{D}$ layers, the crystal exhibits a spontaneous rippling that makes the material structurally anisotropic. We show that rippling comes together with an inhomogeneous in-plane strain profile and anisotropic electrical and optical properties. Using a simple theoretical model, we show that this symmetry breakdown results from a spatial modulation of the van der Waals interaction between layers due to $\mathrm{H}-\mathrm{Q}$ lattice incommensurability. Franckeite is thus a clear example of a natural superlattice system where the interaction between the constituent stacked layers give rise to new properties (anisotropy) not present in the individual 2D constituents (isotropic).

\section{RESULTS AND DISCUSSIONS}

Franckeite flakes have been isolated by mechanical exfoliation of bulk natural franckeite mineral (San José mine, Oruro, Bolivia) with Nitto tape and transferred to other substrates with Gel-film (by Gel-pak) using an all-dry transfer technique. ${ }^{22}$ We point the reader to ref 13 for a detailed characterization of the franckeite bulk crystal.

Figure 1a shows an optical microscopy image of a franckeite flake, whose crystal structure is represented in the inset of Figure $1 \mathrm{a}$, which has been transferred onto a holey $\mathrm{Si}_{3} \mathrm{~N}_{4}$ transmission electron microscopy (TEM) grid. Figure $1 \mathrm{~b}$ is a lowmagnification TEM image acquired on a freely suspended part of the flake shown in Figure 1a, and Figure $1 \mathrm{c}$ is the corresponding high-resolution image. Defocus was optimized to increase the contrast from a striped pattern which is superimposed on the atomic lattice and it can also be seen in

Received: November 2, 2019

Revised: January 3, 2020

Published: January 13, 2020 

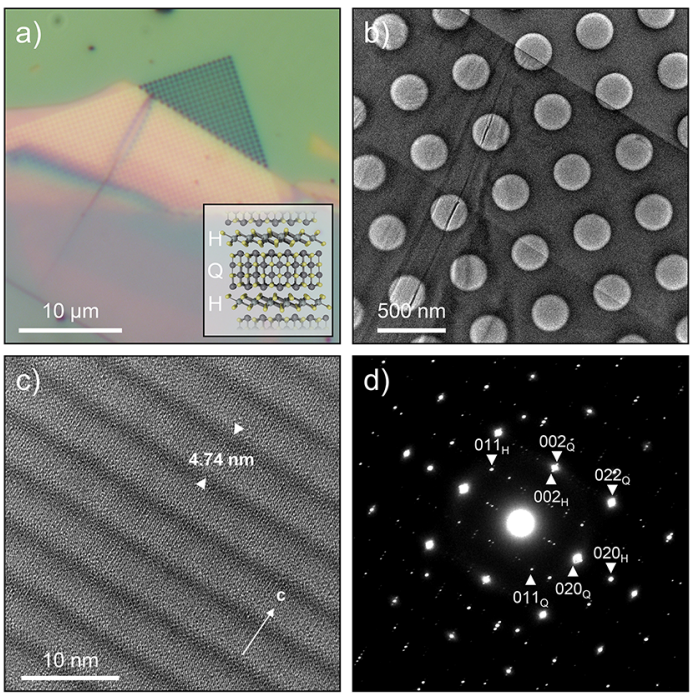

Figure 1. TEM characterization of the structure of franckeite superlattice. (a) Optical image of a franckeite flake over a holey $\mathrm{Si}_{3} \mathrm{~N}_{4}$ TEM support. Inset: crystal structure of franckeite. (b) Lowmagnification HRTEM image of the same franckeite flake. (c) Atomic resolution HRTEM image of the same flake down the (100) direction. (d) Selected area diffraction pattern (SAED) depicting the reflections of both alternating $\mathrm{SnS}_{2}$-like pseudohexagonal $(\mathrm{H})$ and $\mathrm{PbS}$-like pseudotetragonal $(\mathrm{Q})$ lattices.

the selected area diffraction pattern (SAED) in Figure 1d. The SAED exhibits the reflections of the $\mathrm{H}$ and $\mathrm{Q}$ layers along with rows of weak superlattice spots along the $c$-direction due to the striped pattern. A direct comparison between panels $(a-d)$ allows us to determine that the stripes in the franckeite flake are parallel to the flat edge shown in Figure 1a,b.

The striped pattern has been previously observed by Williams and Hyde and it has been found to arise from an out-of-plane deformation of the lattice, which develops periodic ripples with a period of $\sim 4.7 \mathrm{~nm}$ and a peak-to-peak amplitude of $\sim 0.08-0.14$ nm out-of-plane along the franckeite $c$-axis. ${ }^{17,23-25}$ The origin of this periodic rippling is still a subject of debate.

Careful inspection of the structural deformations giving rise to the periodic striped pattern was carried out by strain analysis. Figure 2a shows a high-magnification HRTEM image of a thin region of the franckeite flake in which we performed the analysis (see Materials and Methods). We use the whole field of view within the analyzed image as reference for the strain analysis so the values obtained are expressed with respect to the mean lattice spacing of the analyzed region. The strain tensor $\varepsilon$ component along the $c$-direction, shown in Figure $2 \mathrm{~b}$, depicts strong alternating in-plane expansive and compressive strained regions, which are evidenced in the average profile in Figure 2c. The periodicity of the spatially modulated in plane strain is 4.77 $\mathrm{nm}$ which is in good agreement with the period of the ripple pattern found by direct inspection on the HRTEM image in Figure 1c. This finding suggests that the periodic rippling not only originates from an out-of-plane deformation of the lattice, as previously believed but also from a strong in-plane strain modulation.

In order to get a further insight into the origin of this striped pattern and the strong spatially modulated strain, we have employed a theoretical model, developed in detail in the Supporting Information, that describes, within a continuum medium approximation, how rippling arises from the competi-
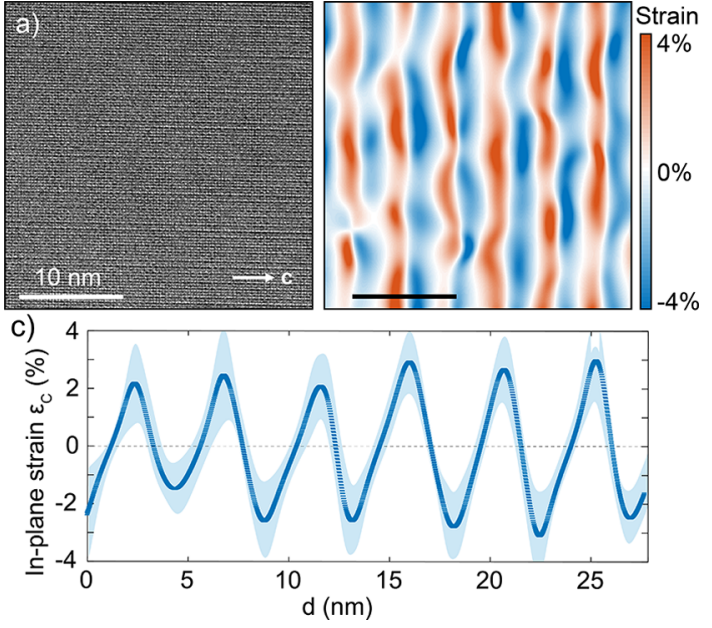

Figure 2. Strain profile analysis of franckeite superlattice. (a) HRTEM image of a franckeite flake down the (100) direction. (b) In-plane strain map along the rippling direction ( $\varepsilon_{\mathrm{c}}$ component of the strain tensor) obtained from the HRTEM image in (a) by geometric phase analysis (GPA). (c) Averaged in-plane strain $\varepsilon_{\mathrm{c}}$ profile, calculated by integrating the strain map of panel (b) along the vertical direction, depicting the periodic expansive and compressive strain modulations along the rippling direction. The shaded area represents the scattering from the mean strain value given by the standard deviation.

tion between inhomogeneous van der Waals interlayer interactions and inhomogeneous elastic deformations. The van der Waals adhesion energy between the $H$ and $Q$ layers is position dependent, following the interlayer moire pattern, as it is controlled by the local atomic alignment between layers, which in turn depends on any inhomogeneous elastic deformations in the material. As the crystal relaxes mechanically to minimize the total energy (adhesion plus elastic), it develops both strong in-plane strains and a small out-of-plane rippling, modulated along the armchair direction of the $\mathrm{H}$ layer. The theoretical results are in quantitative agreement with the experimental observations. Figure 3 a shows a typical lattice structure after relaxation. Figure $3 \mathrm{~b}$ shows the adhesion potential between the hexagonal layers and the bottom/top tetragonal layers. Figure $3 \mathrm{c}, \mathrm{d}$ shows the profiles of ripples $h(y)$, in-plane deformations $u_{y}(y)$, and in-plane strain $\varepsilon_{c}(y)=\mathrm{d} u_{y} / \mathrm{d} y$. The latter exhibits a period that is halved with respect to the ripple profile in agreement with our observations. These results clearly point to adhesion moiré patterns as the most likely origin for franckeite rippling and the observed strong in-plane strains. The relaxation of strain that induces a spatial modulation in the franckeite natural heterostructure belongs to a family of similar phenomena in van der Waals incommensurate lattices. ${ }^{26-28}$ However, franckeite constitutes a unique case because the buckling instability is rather different from the corrugation effects in graphene/hBN and similar systems.

This periodic pattern of lattice strain can be the seed for further anisotropic optical and electrical properties. We first studied the anisotropy in the optical properties by measuring differential reflectance (a magnitude that is proportional to the absorption, see the Supporting Information) upon incidence with linearly polarized light. ${ }^{29}$ Figure 4 a shows some differential reflectance spectra, acquired for the same flake studied by TEM in Figure 1, as a function of the rotation angle of the linear polarizer. The spectra have been labeled with the angle formed between the striped pattern (determined from the TEM images) 
a)
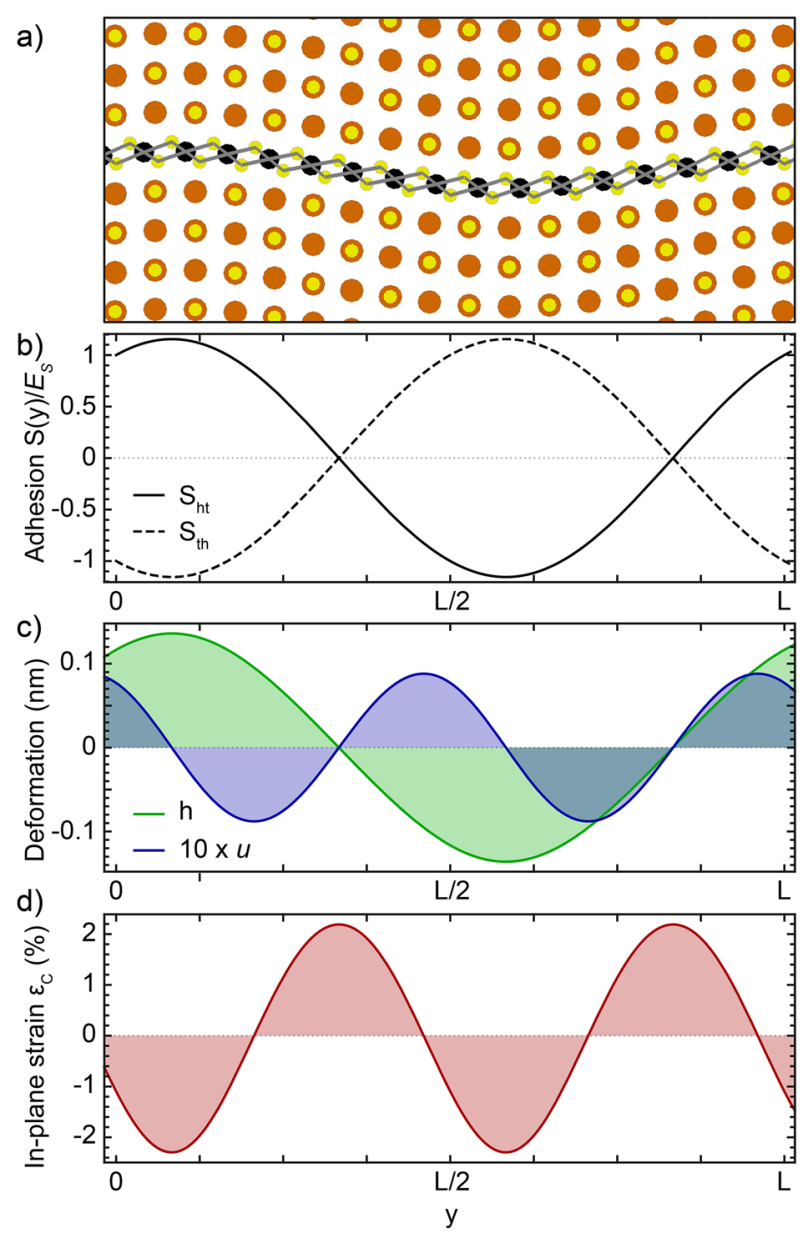

Figure 3. Theoretical model to explain the symmetry breakdown in frankeite. (a) Side view of the computed equilibrium structure of Franckeite across a ripple with $\mathrm{Pb}$ in orange, $\mathrm{S}$ in yellow, and $\mathrm{Sn}$ in black. The shear modulus $\mathrm{G}$ and the $2 \mathrm{D}$ Young modulus $E$ are chosen so that $G V / L E_{S}=128$ and $E V / L E_{S}=45$, where $E_{S}=-E_{S^{\prime}}$ is the maximal adhesion, $V$ is the sample volume, and $L$ is the ripple period; see Supporting Information for details on the theory. (b) Adhesion energy density between hexagonal layer and the tetragonal layers immediately below $\left(S_{\mathrm{ht}}\right)$ and above $\left(S_{\mathrm{th}}\right)$, normalized to the maximum adhesion $E_{\mathrm{S}}$. (c) Equilibrium deformations out of plane $(h(y)$, green) and in-plane $\left(u_{y}(y)\right.$, blue $)$. (d) Equilibrium in-plane strains $\varepsilon_{\mathrm{c}}=\mathrm{d} u_{y} / \mathrm{d} y$, which show variations around $4 \%$ peak-to-peak. and the linearly polarized light (with $0^{\circ}$ corresponding to linearly polarized light along the striped pattern and $90^{\circ}$ linearly polarized light perpendicular to the stripes). Figure $4 \mathrm{~b}$ shows the differential reflectance magnitude extracted at a fixed illumination wavelength $(496 \mathrm{~nm}, 2.5 \mathrm{eV})$ as a function of the angle between the stripes and the linear polarization of the incident light. The data shows a clear angular dependence which is also evident from the polar plot in Figure $4 c$, indicating that franckeite shows linear dichroism (i.e., absorption dependent on the orientation of incident linearly polarized light) even though the individual $\mathrm{H}$ and $\mathrm{Q}$ layers are not expected to show this inplane anisotropy. Apart from the periodical in-plane strain, which is known to strongly modulate the band gap in $2 \mathrm{D}$ materials, $^{30-32}$ the moire pattern also could induce some anisotropy through its effect on the electronic band structure. Nevertheless, we do not expect an important contribution from the moire modulation, since it modulates the electronic structure along both principal axes in the plane and thus it is not strongly anisotropic.

We further study the in-plane symmetry of the electronic properties by fabricating a nanodevice with electrodes designed to address the electrical transport along different crystal orientations (see the optical microscopy image in Figure 5a
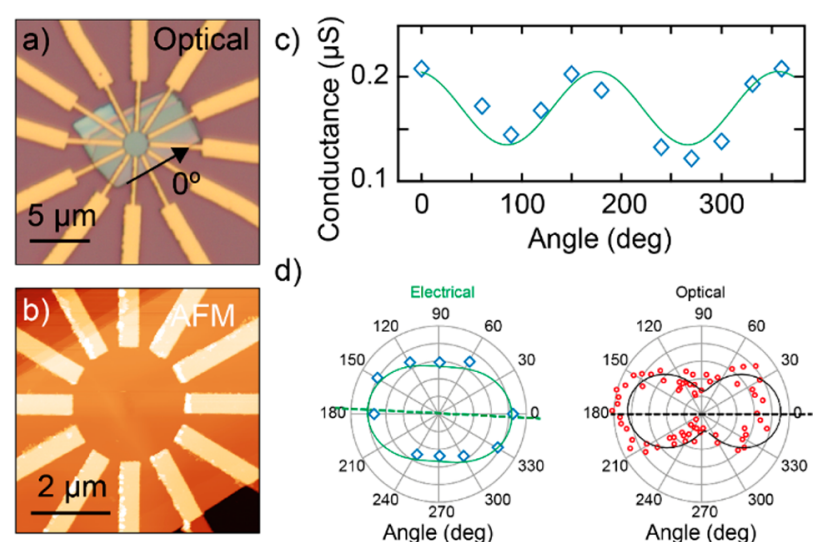

d)

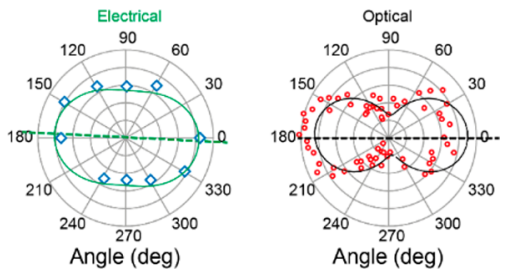

Figure 5. In-plane anisotropy of electrical properties of franckeite. (a,b) Optical image (a) and AFM topography (b) of a franckeite flake over a $\mathrm{SiO}_{2} / \mathrm{Si}$ substrate contacted with $\mathrm{Au}$ electrodes. (c) Conductance recorded between different pairs of electrodes. (d) Conductance (left) and differential reflectance recorded on a Gel-Film substrate prior its transfer to $\mathrm{SiO}_{2} / \mathrm{Si}$ (right) of the franckeite flake shown in polar coordinates.
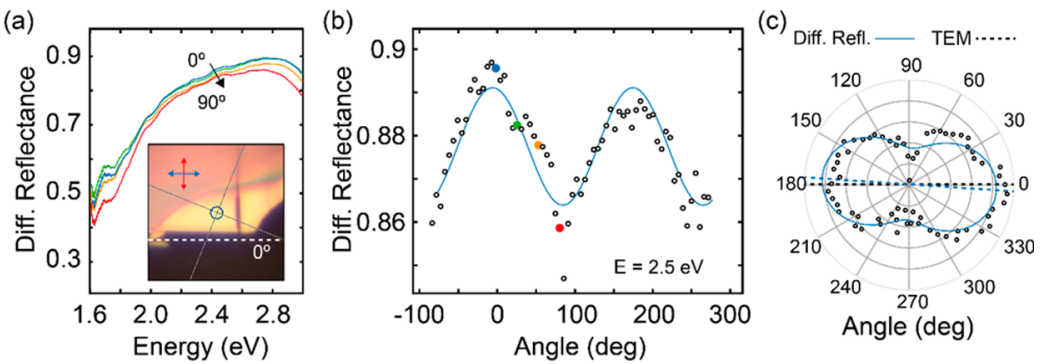

Figure 4. Linear dichroism of frankeite. (a) Differential reflectance spectrum recorded as a function of the rotation angle of the linear polarizer. Inset: optical image of the franckeite flake over a Gel-film substrate prior to its transfer to the TEM grid. (b) Differential reflectance at an energy of $2.5 \mathrm{eV}$ (wavelength $496 \mathrm{~nm}$ ) recorded as a function of the incident light linear polarization angle. (c) Same as (b) shown in polar coordinates. The dashed lines indicate the direction of the maximum of reflectance/absorbance (blue) and the direction of the ripples extracted from the HRTEM image of Figure 1 (black). 


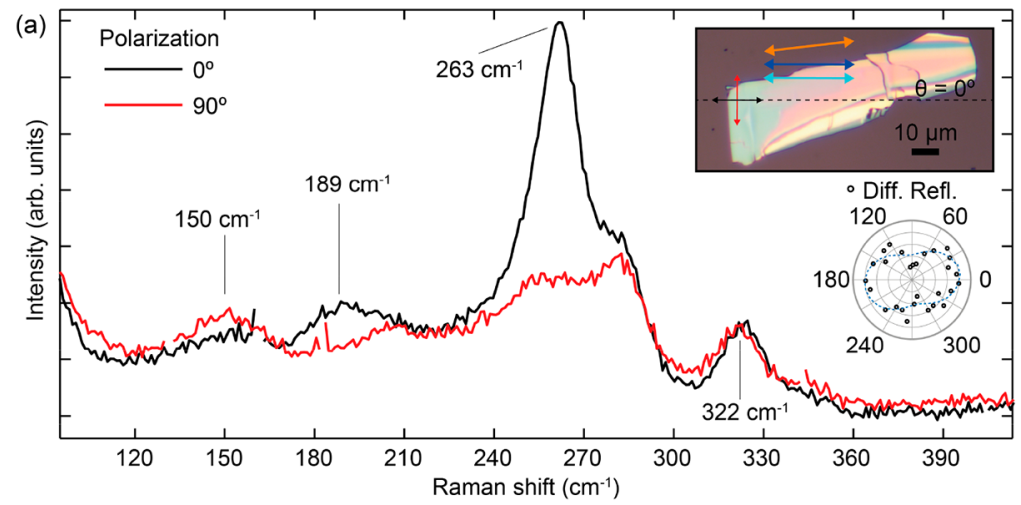

(b)
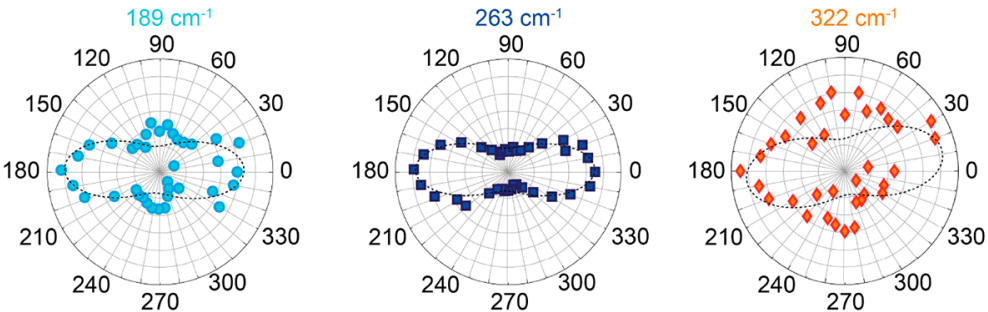

Figure 6. Anisotropic Raman spectra. (a) Polarized Raman spectra with characteristic Raman modes from the $Q$ and $H$ phases. Inset: optical micrograph of the flake used for Raman measurements. The black dashed line marks the direction $\theta=0^{\circ}$, parallel to the ripples. Arrows indicate the directions along which the intensity of the characteristic Raman modes is the highest. (b) Polar plots showing the area of the fitted peaks as a function of $\theta$ for three characteristic modes.

and the topography of the device in Figure $5 b$ ). Figure 5c shows the conductance at different crystal orientations (note that the transport parallel to the long edge of the crystal has been labeled as $0^{\circ}$ ). The electrical conductivity varies by almost a factor of 2 from the transport at $0^{\circ}$ (parallel to the long edge of the flake) to the transport at $90^{\circ}$ (perpendicular to the long edge of the flake) and it follows a sinusoidal relationship that becomes even more evident in the elliptical shape of the data in the polar plot. In order to correlate the electrical measurements with the crystal structure, we performed microreflectance measurements on the same franckeite flake before transferring it to the $\mathrm{SiO}_{2} / \mathrm{Si}$ substrate in a similar way as discussed for Figure 4. Interestingly, from the microreflectance measurements we infer that the long edge of the flake shown in Figure 5a is parallel to the striped pattern of franckeite. Therefore, one can conclude that the conductivity of the device is maximum along the crystal direction parallel to the stripes. Such an increase in conductance could be explained by an increase in the scattering rate experienced by the charge carriers traveling in the direction perpendicular to the ripples, which could result in a decrease of the mobility along that direction. The anisotropic electrical conductivity would also explain the observed linear dichroism: the flakes react to linearly polarized incident light similarly to a polaroid film or a wire-grid polarizer (incident light polarized parallel to the highly conductive crystal axis leads to a higher excitation of charge carriers and thus stronger dissipation of $E$ field).

The in-plane anisotropy of the crystal is also naturally revealed by Raman spectroscopy performed using linearly polarized light. In this case, some of the vibrational modes exhibit a strong increase of the intensity for the light polarized along one of the anisotropy axes. We have performed polarization-resolved Raman measurements in the copolarized configuration, namely for parallel polarization of the excitation and scattered light, and as a function of the angle between the polarization axis and the orientation of the crystal. A typical set of polarized Raman spectra obtained at two different angles is shown in Figure 6a. Several peaks observed in our spectra are in agreement with previously reported data. ${ }^{13,14,18}$ Peaks at 189 and $322 \mathrm{~cm}^{-1}$ correspond to vibrational modes of the hexagonal layer of $\mathrm{SnS}_{2}{ }^{33,34}$ The peak at $263 \mathrm{~cm}^{-1}$ (at polarization $0^{\circ}$ ) and the shoulder at $282 \mathrm{~cm}^{-1}$ can be assigned to the vibrations of $\mathrm{Sb}_{2} \mathrm{~S}_{3}$; however the energies of these modes are lower than in isolated stibnite. $^{35,36}$ This difference is attributed to the complex nature of the tetragonal layer which also contains $\mathrm{Pb}$ and $\mathrm{Sn}$ atoms. Finally, the low energy peak at $150 \mathrm{~cm}^{-1}$ can be related to the vibrations of the $\mathrm{PbS}$ or $\mathrm{SnS}_{2}$ lattices. ${ }^{18}$ The angle dependence of the intensity of the Raman peaks in copolarized configuration (extracted from the fit of the Lorentzian function) is presented in Figure 6b. All the Raman modes exhibit preferential direction with two-fold rotation symmetry characteristic for anisotropic crystals such as black phosphorus, ${ }^{37}$ titanium trisulfide, ${ }^{38}$ or rhenium dichalcogenides. ${ }^{39}$ The angle dependence of the intensity of the Raman modes can be used to determine the orientation of the crystal axes. This is schematically presented in the inset of Figure 6a, where an optical micrograph of the flake together with arrows indicating the directions along which particular Raman modes reach maximum intensity. A dashed line indicates the direction $\theta=0^{\circ}$ parallel to the ripples. The modes around 189,263 , and $322 \mathrm{~cm}^{-1}$ reach maximum intensity when light is polarized perpendicular to the ripple direction, providing an additional tool to determine crystal lattice orientation.

\section{CONCLUSIONS}

In summary, we studied a naturally occurring van der Waals superlattice (franckeite) that clearly illustrates how new properties, absent in the individual constituent layers, emerge in the ensemble system. In particular, franckeite shows a periodic ripple structure in the TEM images that we could reproduce by 
our analytical continuum elasticity model. From the modeling, we learned that a balance between the van der Waals mediated adhesion and the young/shear modulus of the individual layers yields not only an out-of-plane deformation (ripple) but also a simultaneous in-plane strain modulation. We further studied how this strong anisotropy, not present in the individual stacked constituents of the superlattice, affects the electrical and optical properties. We found that the conductance parallel to the ripples is twice as large as in the perpendicular direction. We believe that this anisotropic electrical conductance is the reason for the linear dichroism that we also observed on these samples: the optical absorption is larger for incoming light with linear polarization parallel to the ripples. We also observe intensity changes of characteristic Raman modes of the $\mathrm{Q}$ and $\mathrm{H}$ phase for different orientations of the excitation and scattered light polarization relative to the ripples. This further confirms the presence of optical in-plane anisotropy of franckeite which breaks the initial lattice symmetry of individual layers.

\section{MATERIALS AND METHODS}

Materials. Bulk natural franckeite mineral crystals (from San José mine, Oruro, Bolivia) have been used to extract thin flakes. The bulk crystals have been exfoliated with Nitto tape (224 SPV) onto a Gel-film (WF x4 6.0 mil, by Gel-pak) substrate.

TEM. HRTEM observations were carried out in an aberration-corrected JEOL JEM-GRAND ARM300cF operated at $120 \mathrm{kV}$ and equipped with a cold field emission gun and a fast Gatan OneView camera. Strain analysis was performed by the geometric phase analysis (GPA) method $^{40}$ using the FRWRtools plugin for Digital Micrograph (www.physics.huberlin.de/en/sem/software/software_frwrtools).

Optical Microscopy. Optical microscopy images have been acquired with an AM Scope BA MET310-T upright metallurgical microscope equipped with an AM Scope MU1803 camera with 18 megapixels. The trinocular of the microscope has been modified to connect it to a fiber-coupled Thorlabs spectrometer (part number: CCS200/M) to perform reflection and transmittance spectral measurements. ${ }^{26}$

Fabrication of Franckeite Nanodevices. Frankeite flakes are isolated from the bulk mineral by mechanical exfoliation with Nitto SPV224 tape and subsequently transferred to a $\mathrm{SiO}_{2} / \mathrm{Si}$ substrate with an all dry transfer method. ${ }^{22}$ We use standard ebeam lithography, e-gun metallization, and lift-off procedures to define the metallic contacts $(5 \mathrm{~nm} \mathrm{Ti} / 50 \mathrm{~nm} \mathrm{Au})$.

Characterization of Nanodevices. The electrical characterization is performed in a Lakeshore Cryogenics probe station at room temperature in vacuum $\left(<10^{-5} \mathrm{mbar}\right)$ using home-built source-meter unit electronics

Raman Spectroscopy. Raman spectra were measured in two polarization configurations in a home-built setup. A $532 \mathrm{~nm}$ line of a CW solid-state laser at $2 \mathrm{~mW}$ power was used for excitation through a $50 \times$ magnification long working distance microscope objective $(\mathrm{NA}=0.55)$. The linear polarization direction in the sample plane was controlled by a rotating a halfwaveplate in the excitation beam, and spectra were acquired in two detection polarization configurations: parallel (copolarization) and perpendicular (cross-polarization) to the polarization of the excitation beam. The signal was dispersed by a $50 \mathrm{~cm}$ long spectrometer equipped with a 1800 grooves per millimeter diffraction grating and detected by a nitrogen-cooled chargecoupled device camera. The measurements were carried out at room temperature in ambient conditions.

\section{ASSOCIATED CONTENT}

\section{Supporting Information}

The Supporting Information is available free of charge at https://pubs.acs.org/doi/10.1021/acs.nanolett.9b04536.

Details of the theoretical model, experimental strain analysis (PDF)

\section{AUTHOR INFORMATION}

\section{Corresponding Authors}

Riccardo Frisenda - Materials Science Factory, Instituto de Ciencia de Materiales de Madrid (ICMM-CSIC), Madrid 28049, Spain; 이이.org/0000-0003-1728-7354; Email: riccardo.frisenda@csic.es

Gabriel Sanchez-Santolino - Materials Science Factory, Instituto de Ciencia de Materiales de Madrid (ICMM-CSIC), Madrid 28049, Spain; 이 orcid.org/0000-0001-8036-707X; Email: gsanchezsantolino@ucm.es

Paulina Plochocka - Laboratoire National des Champs Magnétiques Intenses, UPR 3228, CNRS-UGA-UPS-INSA, UPR 3228, CNRS-UGA-UPS-INSA, Grenoble, Toulouse 31400, France; 이이이.org/0000-0002-4019-6138; Email: paulina.plochocka@lncmi.cnrs.fr

Pablo San-Jose - Materials Science Factory, Instituto de Ciencia de Materiales de Madrid (ICMM-CSIC), Madrid 28049, Spain; Email: pablo.sanjose@csic.es

Andres Castellanos-Gomez - Materials Science Factory, Instituto de Ciencia de Materiales de Madrid (ICMM-CSIC), Madrid 28049, Spain; (1) orcid.org/0000-0002-3384-3405; Email: Andres.castellanos@csic.es

\section{Authors}

Nikos Papadopoulos - Kavli Institute of Nanoscience, Delft University of Technology, Delft 2628 CJ, The Netherlands; (i) orcid.org/0000-0002-9972-699X

Joanna Urban - Laboratoire National des Champs Magnétiques Intenses, UPR 3228, CNRS-UGA-UPS-INSA, UPR 3228, CNRS-UGA-UPS-INSA, Grenoble, Toulouse 31400, France

Michal Baranowski - Laboratoire National des Champs Magnétiques Intenses, UPR 3228, CNRS-UGA-UPS-INSA, UPR 3228, CNRS-UGA-UPS-INSA, Grenoble, Toulouse 31400, France; Department of Experimental Physics, Faculty of Fundamental Problems of Technology, Wroclaw University of Science and Technology, Wroclaw 50-370, Poland; 이이.org/ 0000-0002-5974-0850

Alessandro Surrente - Laboratoire National des Champs Magnétiques Intenses, UPR 3228, CNRS-UGA-UPS-INSA, UPR 3228, CNRS-UGA-UPS-INSA, Grenoble, Toulouse 31400, France

Duncan K. Maude - Laboratoire National des Champs Magnétiques Intenses, UPR 3228, CNRS-UGA-UPS-INSA, UPR 3228, CNRS-UGA-UPS-INSA, Grenoble, Toulouse 31400, France

Mar Garcia-Hernandez - Materials Science Factory, Instituto de Ciencia de Materiales de Madrid (ICMM-CSIC), Madrid 28049, Spain

Herre S. J. van der Zant - Kavli Institute of Nanoscience, Delft University of Technology, Delft 2628 CJ, The Netherlands; (1) orcid.org/0000-0002-5385-0282

Complete contact information is available at:

https://pubs.acs.org/10.1021/acs.nanolett.9b04536 


\section{Author Contributions}

${ }^{\#}$ R,F. and G.S.-S. contributed equally to this work.

\section{Author Contributions}

R.F. fabricated the samples and carried out the differential reflectance measurements. G.S.-S. carried out the electron microscopy measurements. N.P. fabricated the electronic devices and carried out the electrical measurements. J.U., M.B., A.S., D.K.M., and P.P. carried out the Raman analysis. M.G.H. and H.S.J.v.d.Z. discussed the electron microscopy and the electronic transport measurements. P.S.J. developed the theoretical model. G.S.S., P.P., P.S.J. and A.C.G. wrote the manuscript with inputs of all other coauthors.

\section{Funding}

European Research Council (ERC): European Union's Horizon 2020 research and innovation program (Grant Agreement 755655, ERC-StG 2017 project 2D-TOPSENSE) EU Graphene Flagship funding (Grant Graphene Core 2, 785219) Spanish Ministry of Economy, Industry and Competitiveness: Juan de la Cierva-formación 2017 FJCI-2017-32919 MINECO: Juan de la Cierva 2015 program FJCI-2015-25427 and FIS2015-65706-P

\section{Notes}

The authors declare no competing financial interest.

\section{ACKNOWLEDGMENTS}

Electron microscopy observations were carried out at the Centro Nacional de Microscopia Electronica, CNME-UCM. This project has received funding from the European Research Council (ERC) under the European Union's Horizon 2020 research and innovation programme (Grant Agreement 755655, ERC-StG 2017 project 2D-TOPSENSE). A.C.G. acknowledges funding from the EU Graphene Flagship funding (Grant Graphene Core 2, 785219). R.F. acknowledges support from the Spanish Ministry of Economy, Industry, and Competitiveness through a Juan de la Cierva-formación fellowship 2017 FJCI-2017-32919. G.S.-S. acknowledges financial support from MINECO (Juan de la Cierva 2015 program, FJCI-2015-25427). P.S.J. acknowledges support from MINECO (FIS2015-65706P). We acknowledge support of the publication fee by the CSIC Open Access Publication Support Initiative through its Unit of Information Resources for Research (URICI).

\section{REFERENCES}

(1) Novoselov, K. S.; Geim, A. K.; Morozov, S. V.; Jiang, D.; Zhang, Y.; Dubonos, S. V.; Grigorieva, I. V.; Firsov, A. A. Electric field effect in atomically thin carbon films. Science 2004, 306 (5696), 666-669.

(2) Novoselov, K.; Jiang, D.; Schedin, F.; Booth, T.; Khotkevich, V.; Morozov, S.; Geim, A. Two-dimensional atomic crystals. Proc. Natl. Acad. Sci. U. S. A. 2005, 102 (30), 10451-10453.

(3) Liu, Y.; Weiss, N. O.; Duan, X.; Cheng, H.-C.; Huang, Y.; Duan, X. Van der Waals heterostructures and devices. Nature Reviews Materials 2016, 1, 16042.

(4) Geim, A. K.; Grigorieva, I. V. Van der Waals heterostructures. Nature 2013, 499 (7459), 419-25.

(5) Novoselov, K.; Mishchenko, A.; Carvalho, A.; Neto, A. C. 2D materials and van der Waals heterostructures. Science 2016, 353 (6298), aac9439.

(6) Jariwala, D.; Marks, T. J.; Hersam, M. C. Mixed-dimensional van der Waals heterostructures. Nat. Mater. 2017, 16, 170-181.

(7) Caldwell, J. D.; Novoselov, K. S. Van der Waals heterostructures: mid-infrared nanophotonics. Nat. Mater. 2015, 14 (4), 364.

(8) Wang, X.; Xia, F. Van der Waals heterostructures: Stacked 2D materials shed light. Nat. Mater. 2015, 14 (3), 264.

(9) Dean, C. R.; Young, A. F.; Meric, I.; Lee, C.; Wang, L.; Sorgenfrei, S.; Watanabe, K.; Taniguchi, T.; Kim, P.; Shepard, K. L. Boron nitride substrates for high-quality graphene electronics. Nat. Nanotechnol. 2010, 5 (10), 722-726.

(10) Frisenda, R.; Navarro-Moratalla, E.; Gant, P.; De Lara, D. P.; Jarillo-Herrero, P.; Gorbachev, R. V.; Castellanos-Gomez, A. Recent progress in the assembly of nanodevices and van der Waals heterostructures by deterministic placement of $2 \mathrm{D}$ materials. Chem. Soc. Rev. 2018, 47, 53-68.

(11) Frisenda, R.; Castellanos-Gomez, A. Robotic assembly of artificial nanomaterials. Nat. Nanotechnol. 2018, 13 (6), 441.

(12) Masubuchi, S.; Morimoto, M.; Morikawa, S.; Onodera, M.; Asakawa, Y.; Watanabe, K.; Taniguchi, T.; Machida, T. Autonomous robotic searching and assembly of two-dimensional crystals to build van der Waals superlattices. Nat. Commun. 2018, 9 (1), 1413.

(13) Molina-Mendoza, A. J.; Giovanelli, E.; Paz, W. S.; Niño, M. A.; Island, J. O.; Evangeli, C.; Aballe, L.; Foerster, M.; Van Der Zant, H. S.; Rubio-Bollinger, G. Franckeite as a naturally occurring van der Waals heterostructure. Nat. Commun. 2017, 8, 14409.

(14) Velický, M.; Toth, P. S.; Rakowski, A. M.; Rooney, A. P.; Kozikov, A.; Woods, C. R.; Mishchenko, A.; Fumagalli, L.; Yin, J.; Zólyomi, V. Exfoliation of natural van der Waals heterostructures to a single unit cell thickness. Nat. Commun. 2017, 8, 14410.

(15) Prando, G. Van der Waals heterostructures: The natural way. Nat. Nanotechnol. 2017, 12 (3), 191-191.

(16) Niu, Y.; Villalva, J.; Frisenda, R.; Sanchez-Santolino, G.; RuizGonzález, L.; Pérez, E. M.; García-Hernández, M.; Burzurí, E.; Castellanos-Gomez, A. Mechanical and liquid phase exfoliation of cylindrite: a natural van der Waals superlattice with intrinsic magnetic interactions. 2D Mater. 2019, 6 (3), No. 035023.

(17) Williams, T.; Hyde, B. Electron microscopy of cylindrite and franckeite. Phys. Chem. Miner. 1988, 15 (6), 521-544.

(18) Ray, K.; Yore, A. E.; Mou, T.; Jha, S.; Smithe, K. K.; Wang, B.; Pop, E.; Newaz, A. Photoresponse of natural van der Waals heterostructures. ACS Nano 2017, 11 (6), 6024-6030.

(19) Gusmão, R.; Sofer, Z.; Luxa, J.; Pumera, M. Layered franckeite and teallite intrinsic heterostructures: shear exfoliation and electrocatalysis. J. Mater. Chem. A 2018, 6 (34), 16590-16599.

(20) Burzurí, E.; Vera-Hidalgo, M.; Giovanelli, E.; Villalva, J.; Castellanos-Gomez, A.; Pérez, E. M. Simultaneous assembly of van der Waals heterostructures into multiple nanodevices. Nanoscale 2018, 10 (17), 7966-7970.

(21) Gant, P.; Ghasemi, F.; Maeso, D.; Munuera, C.; López-Elvira, E.; Frisenda, R.; De Lara, D. P.; Rubio-Bollinger, G.; Garcia-Hernandez, M.; Castellanos-Gomez, A. Optical contrast and refractive index of natural van der Waals heterostructure nanosheets of franckeite. Beilstein J. Nanotechnol. 2017, 8 (1), 2357-2362.

(22) Castellanos-Gomez, A.; Buscema, M.; Molenaar, R.; Singh, V.; Janssen, L.; van der Zant, H. S.; Steele, G. A. Deterministic transfer of two-dimensional materials by all-dry viscoelastic stamping. $2 D$ Mater. 2014, 1 (1), No. 011002.

(23) Wang, S.; Kuo, K. Crystal lattices and crystal chemistry of cylindrite and franckeite. Acta Crystallogr., Sect. A: Found. Crystallogr. 1991, 47 (4), 381-392.

(24) Makovicky, E.; Petř́íček, V.; Dušek, M.; Topa, D. The crystal structure of franckeite, $\mathrm{Pb} 21.7 \mathrm{Sn} 9.3 \mathrm{Fe} 4.0 \mathrm{Sb} 8$. 1S56. 9. Am. Mineral. 2011, 96 (11-12), 1686-1702.

(25) Henriksen, R. B.; Makovicky, E.; Stipp, S.; Nissen, C.; Eggleston, C. M. Atomic-scale observations of franckeite surface morphology. Am. Mineral. 2002, 87 (10), 1273-1278.

(26) Carr, S.; Fang, S.; Zhu, Z.; Kaxiras, E. Exact continuum model for low-energy electronic states of twisted bilayer graphene. Physical Review Research 2019, 1 (1), No. 013001.

(27) Carr, S.; Massatt, D.; Torrisi, S. B.; Cazeaux, P.; Luskin, M.; Kaxiras, E. Relaxation and domain formation in incommensurate twodimensional heterostructures. Phys. Rev. B: Condens. Matter Mater. Phys. 2018, 98 (22), 224102.

(28) Kumar, H.; Er, D.; Dong, L.; Li, J.; Shenoy, V. B. Elastic deformations in $2 \mathrm{D}$ van der Waals heterostructures and their impact on optoelectronic properties: predictions from a multiscale computational approach. Sci. Rep. 2015, 5, 10872. 
(29) Frisenda, R.; Niu, Y.; Gant, P.; Molina-Mendoza, A. J.; Schmidt, R.; Bratschitsch, R.; Liu, J.; Fu, L.; Dumcenco, D.; Kis, A.; Lara, D. P. d.; Castellanos-Gomez, A. Micro-reflectance and transmittance spectroscopy: a versatile and powerful tool to characterize $2 \mathrm{D}$ materials. J. Phys. D: Appl. Phys. 2017, 50 (7), No. 074002.

(30) Ram, B.; Singh, A. K. Strain-induced indirect-to-direct band-gap transition in bulk SnS 2. Phys. Rev. B: Condens. Matter Mater. Phys. 2017, 95 (7), No. 075134.

(31) Li, H.; Castelli, I. E.; Thygesen, K. S.; Jacobsen, K. W. Strain sensitivity of band gaps of Sn-containing semiconductors. Phys. Rev. B: Condens. Matter Mater. Phys. 2015, 91 (4), No. 045204.

(32) Rabii, S. Investigation of energy-band structures and electronic properties of $\mathrm{PbS}$ and PbSe. Phys. Rev. 1968, 167 (3), 801.

(33) Smith, A.; Meek, P.; Liang, W. Raman scattering studies of SnS2 and SnSe2. J. Phys. C: Solid State Phys. 1977, 10 (8), 1321.

(34) Mead, D.; Irwin, J. Raman spectra of SnS2 and SnSe2. Solid State Commun. 1976, 20 (9), 885-887.

(35) Kharbish, S.; Libowitzky, E.; Beran, A. Raman spectra of isolated and interconnected pyramidal XS3 groups $(\mathrm{X}=\mathrm{Sb}, \mathrm{Bi})$ in stibnite, bismuthinite, kermesite, stephanite and bournonite. Eur. J. Mineral. 2009, 21 (2), 325-333.

(36) Efthimiopoulos, I.; Buchan, C.; Wang, Y. Structural properties of $\mathrm{Sb} 2 \mathrm{~S} 3$ under pressure: evidence of an electronic topological transition. Sci. Rep. 2016, 6, 24246.

(37) Ribeiro, H. B.; Pimenta, M. A.; De Matos, C. J.; Moreira, R. L.; Rodin, A. S.; Zapata, J. D.; De Souza, E. A.; Castro Neto, A. H. Unusual angular dependence of the Raman response in black phosphorus. ACS Nano 2015, 9 (4), 4270-4276.

(38) Island, J. O.; Biele, R.; Barawi, M.; Clamagirand, J. M.; Ares, J. R.; Sánchez, C.; van der Zant, H. S.; Ferrer, I. J.; D’Agosta, R.; CastellanosGomez, A. Titanium trisulfide (TiS3): a 2D semiconductor with quasi1D optical and electronic properties. Sci. Rep. 2016, 6, 22214.

(39) Chenet, D. A.; Aslan, O. B.; Huang, P. Y.; Fan, C.; van der Zande, A. M.; Heinz, T. F.; Hone, J. C. In-plane anisotropy in mono-and fewlayer ReS2 probed by Raman spectroscopy and scanning transmission electron microscopy. Nano Lett. 2015, 15 (9), 5667-5672.

(40) Hÿtch, M.; Snoeck, E.; Kilaas, R. Quantitative measurement of displacement and strain fields from HREM micrographs. Ultramicroscopy 1998, 74 (3), 131-146. 